\title{
Utility-based Rate Allocation Scheme for Mobile Video Streaming over Femtocell Networks
}

\author{
Shan Guo Quan*, Jian Xu** and Young Yong Kim*
}

\begin{abstract}
This paper proposes a utility-based data rate allocation algorithm to provide high-quality mobile video streaming over femtocell networks. We first derive a utility function to calculate the optimal data rates for maximizing the aggregate utilities of all mobile users in the femtocell. The total sum of optimal data rates is limited by the link capacity of the backhaul connections. Furthermore, electromagnetic cross-talk poses a serious problem for the backhaul connections, and its influence passes on to mobile users, as well as causing data rate degradation in the femtocell networks. We also have studied a fixed margin iterative water-filling algorithm to achieve the target data rate of each backhaul connection as a counter-measure to the cross-talk problem. The results of our simulation show that the algorithm is capable of minimizing the transmission power of backhaul connections while guaranteeing a high overall quality of service for all users of the same binder. In particular, it can provide the target data rate required to maximize user satisfaction with the mobile video streaming service over the femtocell networks.
\end{abstract}

Keywords: Utility, femtocell network, backhaul, cross-talk, video streaming

\section{Introduction}

Recent advances in high-speed local area wireless networks and video codec with a high compression rate have made mobile video streaming more popular in wireless cellular networks.

According to a recent survey, approximately $30 \%$ of mobile calls are made at home. Typically, a recent Mckinsey report highlighted that $35 \%$ of mobile TV is watched in the home [1]. Since most mobile users are now familiar with using data services inside their homes, the number of potential mobile users who receive mobile video streaming services at home will continue to increase over the next few years. However, there are many areas with poor signal reception as well as coverage holes where the macro-cellular network is unavailable, especially in residential areas, making the delivery of high-speed and reliable mobile data to mobile users in the home a tough challenge for macro-cellular networks.

The femtocell network is a promising solution that supports fast and stable wireless data services to mobile users at a very low cost in the home. This encourages users to use mobile data services over the femtocell networks. Femtocell networks are tiny, low-power $3 \mathrm{G}$ radio systems that can be plugged into residential broadband connections to provide

Manuscript received May 8, 2009; accepted June 26, 2009

Corresponding Author: Shan Guo Quan

* Dept. of Electrical and Electronics Engineering, Yonsei University, Seoul, Korea(sgquan@yonsei.ac.kr,y2k@yonsei.ac.kr)

** MC Lab., LG Electronics, 533, Hogye 1 dong, Dongan-gu, Anyang, Gyeonggi-do, 431-080, Korea(jianxu@yonsei.ac.kr) a mobile signal directly inside the home [19]. Furthermore, these femtocell networks enable the operators to reduce the cost of infrastructure further still and pass these cost savings on to the customers, enabling mobile users to enjoy entertainment and information services (e.g. MTV, YouTube, sports highlight, newscasts, and so forth) more economically in the home.

Although the femtocell networks offer certain advantages, they also present several technical difficulties. For instance, there are problems related with control of the interference, handoff, and co-channel management between the macrocell and femtocell networks [2,3].

Equally importantly, there are obvious backhaul issues when household broadband links (e.g. digital subscriber line [DSL] or cable) are used as the backhaul connections for the femtocell networks. In particular, cross-talk is a major issue with modern DSL systems, and often represents co-channel interference. The influence of cross-talk will be passed on to mobile users in the femtocell networks because the total data rate needed to support mobile users is restricted by the link capacity of backhaul connections. The effects of cross-talk are usually mitigated by static spectrum management (SSM)[11] and dynamic spectrum management (DSM)[12]. Both more recent research $[5,6]$ and earlier research [7-10] have investigated certain scenarios aimed at reducing the effects of cross-talk.

In this paper, spectrum management with regard to backhaul connections in the femtocell network is proposed from the perspective of utility. 'utility' represents the degree of the mobile user's satisfaction, and the utility function is 
defined as the relationship between the video data rate, service cost, and mobile user's satisfaction. To maximize the mobile user's utility, the target data rate of the DSLbased backhaul connection must be greater than or equal to the total sum of the optimal data rates for mobile users over the femtocell networks. Therefore, we have studied a fixed margin (FM) iterative water-filling (IW) algorithm, which can be used to realize much of the gain from the DSLbased backhaul connections.

The remainder of the paper is organized as follows. Section 2 describes the DSL-based backhaul connections in the femtocell network and the system model. In section 3 , we first derive the utility function, which is based on video quality and service cost, by conducting subjective assessments. In section 4, we have studied the FM iterative water-filling algorithm over the DSL-based backhaul connections in the femtocell networks. In section 5, simulation results and system performance analysis are discussed. Finally, the conclusion is presented in section 6 .

\section{The DSL-based Femtocell Networks and System Model}

Fig.1 shows how DSL-based household broadband links are used as backhaul connections in femtocell networks. As the demand for a high data rate increases, we assume that DSL-based backhaul connections are composed of either remote terminal (RT) / central office (CO)-based VDSLs or of an optical network unit (ONU)-based VDSL and ADSL2+[4] mixed system. Each Femto-based transceiver station (Femto-BTS) plugs into a DSL-modem and provides a mobile signal directly, with one Femto-BTS being able to cover a whole home and provide a high data rate because the mobile users are very close to the Femto-BTS within the house. Wireless downlink data transmission-such as

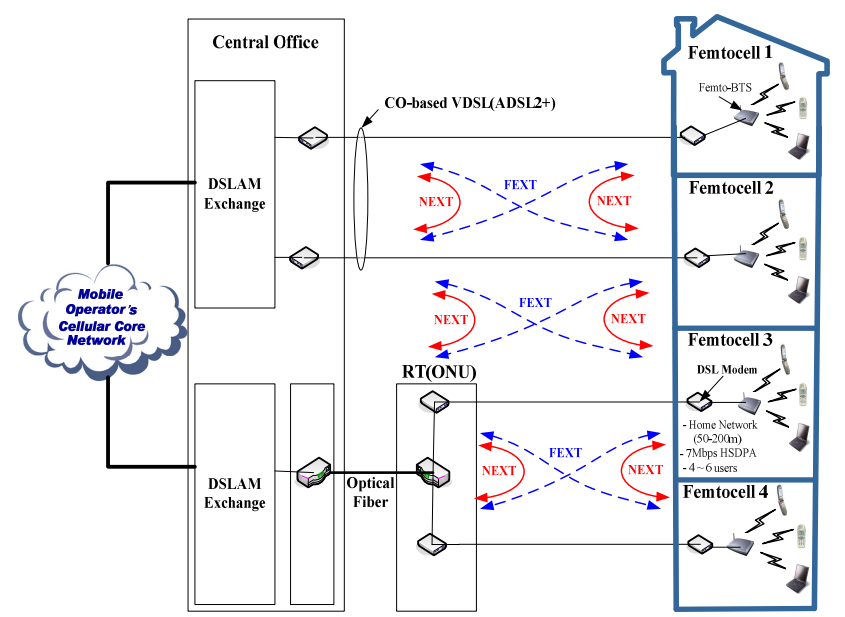

Fig. 1. DSL-based femtocell network architecture high-speed downlink packet access (HSDPA) - is also used. In addition, DSL modems use frequencies above the traditional voice band to carry high-speed data. The telephone channels are extremely frequency selective. One way to combat inter-symbol interference (ISI) is to use discrete multi-tone (DMT) modulation, which divides the frequency band into a large number of ISI-free sub-channels and allows each sub-channel to carry a separate data stream.

This paper focuses on the DMT modulation scheme as standardized for ADSL2+ and VDSL. Since a great number of DSLs are bundled together in the same DSL binder, the multi-lines have electromagnetic interference between each other, and result in two types of cross-talk noises (see Fig. 1): one consists of near end cross-talk (NEXT), which refers to cross-talk created by transmitters located on the same side as the receiver, while the other is far end crosstalk (FEXT), which refers to cross-talk created by transmitters located on the opposite end of the line. NEXT is usually much stronger than FEXT. Usually, in DSL transmission systems, frequency division duplex (FDD) is used to avoid NEXT. Thus, FEXT is the main source of cross-talk. Assuming that the entire system has $n$ frequency tones (sub-channels), the signal-to-interference-plus-noise ratio (SINR) of line $i$ in sub-channel $n$ is expressed as:

$$
\operatorname{SINR}_{i}{ }^{n}=\frac{P_{i}{ }^{n}\left|G_{i, i}\right|^{2}}{\sum_{j \neq i} P_{j}^{n}\left|G_{i, j}^{n}\right|^{2}+N_{i}^{n}}
$$

where $N_{i}^{n}$ and $P_{i}^{n}$ are the background noise power of line $i$ in sub-channel $n$ and the signal power respectively. $\mathrm{G}_{\mathrm{i}, \mathrm{i}}^{\mathrm{n}}$ represents the direct channel gain of line $i$ in subchannel $n$, while $G_{i, j}^{n}$ represents the cross-talk channel gain from line $j$ to line $i$ Assuming that all the transmitted signals and background noises are Guassian, the reliably transmittable bit rate - with QAM modulation under a certain bit error rate (BER) and a coding scheme - is then expressed as:

$$
r_{i}^{n}=\log _{2}\left(1+\frac{S I N R_{i}^{n}}{\Omega}\right)
$$

Using (1), (2) becomes

$$
r_{i}^{n}=\log _{2}\left(1+\frac{P_{i}^{n}\left|G_{i, i}^{n}\right|^{2}}{\Omega \cdot\left(\sum_{j \neq i} P_{j}^{n}\left|G_{i, j}^{n}\right|^{2}+N_{i}^{n}\right)}\right)
$$


where $\Omega$ is referred to as the SINR gap, which is the function of the target BER, noise margin, and coding schemes [13]. For example, $\Omega=9.8+\gamma_{m}-\gamma_{c}$ where $\gamma_{m}$ is the margin, $\gamma_{c}$ is the coding gain of the receiver, and the number 9.8 is to ensure that the BER is equal to $10^{-7}$. The data rate of line $i$ is then

$$
R_{i}=\frac{1}{T_{s}} \cdot \sum_{n=1}^{M} r_{i}^{n}
$$

where $T_{s}$ is a symbol period of packed data. The purpose of the system's design is to maximize the set of rates $\left(R_{1}, \cdots, R_{N}\right)$ subject to the power constraints. A rate region is defined as the union of all the rate sets $\left(R_{1}, \cdots, R_{N}\right)$ that can be achieved while satisfying the following power constraint:

$$
P_{i}=\sum_{n=1}^{M} P_{i}^{n}, P_{i} \leq P_{i}^{\max }, \text { for } i=1, \cdots, N
$$

where $P_{i}$ is the transmission power of line $i$, and $P_{i}^{\max }$ is the maximum transmission power for line $i$.

\section{Utility Function}

Whenever a service provider serves streaming contents at the full rate, not every user can be satisfied. In other words, users wish to receive a service of high quality at low cost. However, a high-quality service is usually supported by high data rate transmission. Moreover, the cost of a service generally increases in proportion to any increase in the data rate. Therefore, optimization between cost and quality is required. If the cost of a service is too high, people will be very slow to adopt it. Femtocell, combined with "femtozone" tariffs, can solve the problem, giving a good user experience in the home at an affordable cost to the operator, with price discounts that don't leak outside the home [19].

Hence, it is evident that service cost and video quality are important factors in guaranteeing a mobile user's satisfaction with a mobile video streaming service. The user's perception of service quality can be determined by user utility. Next, we derived a utility function, which is related to service cost and video quality. We considered the optimal data rate allocation to achieve the maximum aggregate utility for all users. The goal of designing the algorithm is to achieve an optimal system wherein the users' choice (of the data service rates they are willing to pay for) and the network's choice (of the allocated rate) are in equilibrium. In this section, we use utility as the objective of optimization. Here, the parameters in our utility function are 'cost', 'video quality', and 'data rate'. The utility function is derived as follows (each component will be explained later):

$$
U(C, V, D)=U(C) \cdot U(V, D)
$$

where $C$ is the service cost; $V$ is the video quality; and $D$ is the service data rate over the wireless link.

$U(C)$. Depending on the various environments, a wide variety of cost policies can be applied [14]. We assume a simple cost policy in which the cost increases in proportion to the service rate. The underlying motivation for using this type of utility function is as follows: In general, people are discouraged from using a service as the charge increases. Therefore, the cost is in inverse proportion to utility [15]. If we set the maximum value of utility as one, then the utility function of cost is expressed as

$$
U(C)=\left(1-\frac{C}{C_{T h}}\right)+\phi
$$

where $C_{T h}$ represents the cost of the maximum service rate and $\phi$ represents the basic utility. Basic utility means that people who are willing to pay the maximum charge for service exist.

$U(V, D)$ One method of measuring utility has been recommended by ITU-R, whereby the variant 2 of the double-stimulus impairment scale (DSIS) in the ITU-R BT.500-10[16] is used for subjective user-end perceptual quality assessments. The video quality is evaluated by using the terms shown in Fig.2.

The mobile video streaming service is very sensitive to data rate. That being the case, the degree of satisfaction felt by the user is directly affected by the available data rate and video quality. Fig. 2 shows that if the quality of a video service is lower than 0.7 , each mobile user will be annoyed by the bad video quality. Otherwise, users are usually always satisfied with a video streaming service and don't express annoyance. We use a spatial resolution ratio, $V$, to change the video quality. $V$ is defined on condition that the critical video has a rate of $1024(\mathrm{Kbps})$, a playing time of $10 \mathrm{sec}$, and spatial resolution of $640 \times 480$ pixels, as shown in [17]. Improvements in video quality lead to an increase in data size. Videos are compressed in different qualities, and the bit rates are changed proportionally according

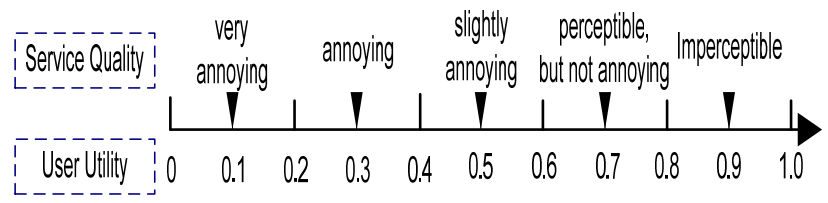

Fig. 2. Relationship between user utility and service quality 
to video quality. From [17], the degree of a user's satisfaction is given by the utility function of the data rate requirement and video quality as follows.

$$
U(V, D)=0.8855 \mathrm{e}^{\left(-\frac{0.1588 V}{D}\right)}\left(1-\mathrm{e}^{-5.032 V}\right)
$$

However, service cost was completely ignored in [17]. As such, we define service cost as follows:

$$
C=\alpha D
$$

where $\alpha$ is a proportional constant. Using (7), (8) and (9), (6) becomes

$$
U(C, V, D)=\left(1-\frac{\alpha D}{C_{T h}}+\phi\right)\left(0.8855 \mathrm{e}^{\left(-\frac{0.1588 V}{D}\right)}\left(1-\mathrm{e}^{-5.032 V}\right)\right)
$$

We formulate the data rate allocation problem as follows:

$$
\begin{array}{ll}
\max & \sum_{x_{r}, r \in N_{f}} U_{r}\left(C, V, x_{r}\right)=\sum_{x_{r}, r \in N_{f}} U_{r}\left(\alpha x_{r}, V, x_{r}\right) \\
\text { s.t. } & \sum_{\mathrm{r} \in N_{f}} x_{r, h} \leq C_{h}^{t} \quad \forall h \in L, \mathrm{t} \in\{A D S L 2+, V D S L\} \\
& x_{r, h}>0 \quad \forall h \in L, \forall r \in N_{f}
\end{array}
$$

where the following are given as inputs to the problem:

$L \quad$ is a set of femtocells.

$N_{f}$ is the number of mobile users in the femtocell.

$C_{h}^{t} \quad$ is the capacity of the t-type backhaul connection for the femtocell $h(h \in L)$.

$U_{r}$ is the utility function of the data rate of the user $r$.

$x_{r, h}$ is the data rate for the user $r$ in the femtocell $h$.

To support an optimal data rate per mobile user, each backhaul connection has to guarantee that the totality of the optimal data rate of the mobile users is less than or equal to the capacity of the backhaul connection in the femtocell. To simplify the analysis, we can assume that the data rate per mobile user is equal to $C_{h}^{t} / N_{f}$ because the received power of the mobile user is more or less the same within the femtocell, which in turn means that the received data rate per user is almost the same. If there are $K$ mobile users in the $t$ type of backhaul connection-based femtocell $h$, the target $C_{h}^{t}$ is equal to $K \times R$, where $R$ is the required data rate per mobile user in the femtocell $h$. If it is assumed that the optimal data rate per user is equal to $R_{\text {optimal }}$ in order to maximize the user's utility, then $C_{h}^{t}=K \times R_{\text {optimal }}$. In the next section, we illustrate how to achieve the target value of $C_{h}^{t}$ at the backhaul connections in order to maximize the utility of the mobile user.

\section{Fixed Margin Iterative Water-Filling Algorithm for Backhaul Connections}

\subsection{Iterative Water-Filling Algorithm for Backhaul Connections}

It is known that water-filling is the optimal-power distribution algorithm for singer-user communication, and provides the basis for the power and bit allocation schemes in most DMT-based modems. Given the channel signal-tonoise ratio (SNR) information in the frequency domain $S N R(f)$, the optimal power allocation $P(f)$ for maximizing the data rate is obtained by allocating more power to frequency bands with higher channel SNR. The single user waterfilling procedure is given in [13]. Iterative water-filling can be viewed as an extension of the water-filling process for a multi-user communication environment. This algorithm is based on the formulation of power allocation in the multiuser interference channel as a non-cooperative game, where each user adjusts his/her power allocation to maximize his/her own data rate, while regarding all other interference as noise. The conditions for the existence and the uniqueness of the Nash Equilibrium were given in [5], and these conditions have been met by experiment for every possible DSL channel environment that has been tested. Thus, an interactive water-filling algorithm, wherein at every step each modem updates its power spectral density (PSD) while regarding all interference as noise, converges to the unique Nash equilibrium from any starting point. The iterative water-filling procedure is expressed in Fig. 3 in accordance with the descriptions given above. Starting with any initial power allocation, the procedure will usually converge to a stationary point after step1 or step2 in practice. In Fig. 3,

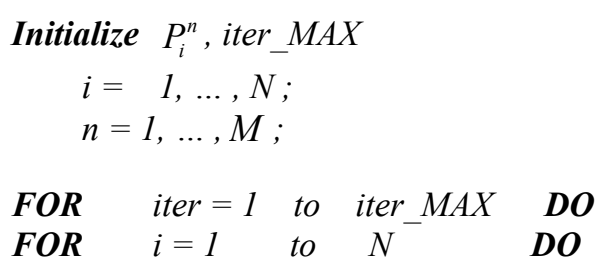

step1: Compute water-filling power $P_{i}$ for line $i$ only, where $P_{i}^{n}$ is held at the previous value for $j \neq i$

step2: Re-compute cross-talk noise power into all other lines

$$
j \neq i,\left(\sigma_{j, i}^{n}\right)^{2}=P_{i}^{n} \cdot\left|G_{j, i}^{n}\right|^{2}
$$

\section{END FOR}

iter $=$ iter +1 ;

\section{END FOR}

Fig. 3. Pseudo code for the iterative water-filling algorithm 
$N$ is the number of lines in the binder, $n$ is a tone index, and $j$ and $i$ are the line indices that go from 1 to $N$. Also, $\left|G_{j, i}^{n}\right|^{2}$ is the transfer function magnitude from line $i$ to line $j$ at tone $n$, and $\left(\sigma_{j, i}^{n}\right)^{2}$ is the cross-talk noise on line $j$ from line $i$ at tone $n$.

\subsection{Fixed Margin Mode}

The FM mode of iterative water-filling, which only uses the amount of power needed to guarantee the best overall use of the binder, is frequently used by service providers who want to ensure that all services offer the best performance possible. There are two reasons to use FM mode: one is that the use of FM mode allows other system that have insufficient margin to improve their performance. The other is that the coordination is unnecessary as long as the attempted data rates are in the achievable rate region of iterative water-filling. Any DSL modem that minimizes the transmission power required at a given fixed margin for a certain probability of BER in order to achieve the service provider's target data rate is said to operate within a fixed margin. The minimization of power consumption by a fixed-margin DSL modem can be achieved theoretically by DSL power allocation $P_{i}^{n}$ at tone $n$, which satisfies the equation

$$
\begin{aligned}
& P_{i}^{n}+\frac{\Omega}{\frac{\left|G_{i, i}^{n}\right|^{2}}{\sum_{j \neq i}\left|G_{i, j}^{n}\right|^{2} P_{j}^{n}+N_{i}^{n}}} \\
& =P_{i}^{n}+\frac{10^{0.1\left(9.8+\gamma_{m}-\gamma_{c}\right)}}{\frac{\left|G_{i, i}^{n}\right|^{2}}{\sum_{j \neq i}\left|G_{i, j}^{n}\right|^{2} P_{j}^{n}+N_{i}^{n}}} \\
& =\text { constant for all used tones }
\end{aligned}
$$

Actually, (12) is the standard form of water-filling in addition to the fixed margin. Thus, for the principle of water-filling, the constant in (12) is determined by allocating non-negative power to those tones with higher SINRs. And also (12) above is solved until the desired data rate of the DSL transmission system is equal to the data rate computed by

$$
\begin{aligned}
R_{i} & =\nabla f \cdot \sum_{n=1}^{M} \log _{2}\left(1+\frac{\operatorname{SINR}_{i}^{n}}{\Omega}\right) \\
& =\nabla f \cdot \sum_{n=1}^{M} \log _{2}\left(1+\frac{\operatorname{SINR}_{i}^{n}}{10^{0.1\left(9.8+\gamma_{m}-\gamma_{c}\right)}}\right)
\end{aligned}
$$

where $\nabla f$ is the frequency range for every tone. Based on this fixed-margin mode, we implement the IW in the multi- lines case in order to obtain minimized power consumption by every line. The performance analysis is shown in the next section.

\section{Simulation and Performance Analysis}

The performance of the fixed-margin IW algorithm is analyzed in this section. Two realistic DSL-based backhaul connections are considered for the downstream in the femtocell: one is an RT/CO-distributed pure VDSL line, the other consists of a mixed system composed of ADSL2+ with CO-distributed lines and ONU-distributed VDSL lines within the same binder (see Fig. 1). Each cell has $4 \sim 6$ mobile users, and each Femto-BTS offers data transfer speeds with a maximum wireless signal rate of up to $7 \mathrm{Mbps}$ based on HSDPA techniques. However, the second phase of HSDPA has been specified in the upcoming 3GPP release 7 and has been named high speed packet access (HSPA) Evolved. It can support data rates of up to 42Mbps. With the evolution of HSPA, long-term evolution (LTE) will offer data rates of over 320Mbps for downlinks [20] in the near future. This implies that short-range, high-datarate transmission for mobile video streaming is not critical in the femtocell, so it can also provide high-definition wireless video streaming. We consider the stationary or quasi-stationary distribution of mobile users in the femtocell. Thus, each mobile user receives data via a quasi-stationary, flat-fading channel environment.

The following example is presented to explain how the algorithm works. Suppose that $\alpha=4, \phi=0.1$, and $C_{T H}=600$. Using (10), Fig. 4 shows that there are optimum data rates to maximize the user's utility. As the value of $V$ increases, the user's utility also increases.

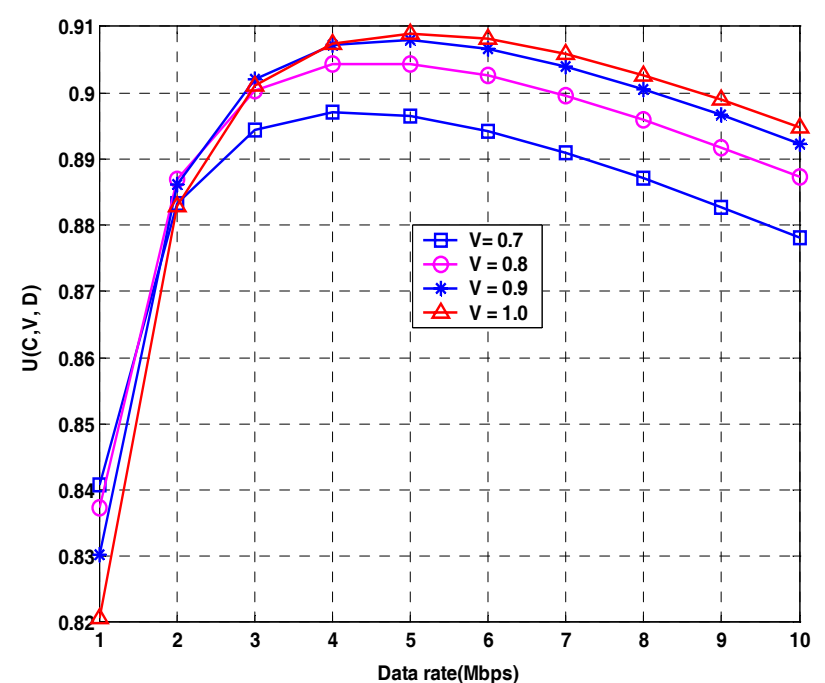

Fig. 4. Utility values for differences in the data rate and video quality 
For example, if there are 4 mobile users in the VDSLbased femtocell and the desired video quality $(V)$ for each mobile user is equal to 0.8 , the optimal data rate per mobile user for maximizing the user's utility will be roughly equal to $5 \mathrm{Mbps}$, as shown in Fig.4. Thus, the target data rate of the VDSL-based backhaul connection is equal to $20 \mathrm{Mbps}$. Then, the IW algorithm aims to achieve the target data rate for the different DSL-based backhaul connections in the femtocell network.

\subsection{ONU-VDSL and ADSL2+ Mixed Backhaul Connections}

We investigated downstream transmission in ADSL2+ with 25 5,000-ft CO-distributed and 25 2,000-ft ONUdistributed VDSL lines. The ONU is located 4000 feet from the $\mathrm{CO}$, as depicted in Fig.5. In order to obtain the rate regions, a maximum transmission power of $20.4 \mathrm{dBm}$ is applied to each CO-based ADSL2+ modem, while $11.5 \mathrm{dBm}$ is the maximum transmission power for the ONU-based VDSL modems [18]. The twisted pairs are assumed to be $26 \mathrm{AWG}$, and the cross-talk transfer functions are computed using the well known FEXT model [4,18].

Fig. 6 shows the rate regions corresponding to the fixedmargin IW algorithm for the CO-based ADSL2+ and ONU-based VDSL downstream lines. Applying traditional SSM techniques, such as in the nominal situation of T1.417 using the 998-standardized spectrum for VDSL of $60 \mathrm{dBm} / \mathrm{Hz}$, and in the new standard for ADSL2+ using the maximum level of PSD [12], one can see from Fig.6 that the rates for the CO-based ADSL2+ lines are considerably lower than the rates of the CO-based ADSL2+ lines, which employ the fixed-margin IW algorithm. In fact, the extended second downstream band in the ADSL2+ line cannot be used in practice because of the strong cross-talk generated by the ONU-based VDSL lines. However, it makes a huge difference if we adopt the fixed-margin IW algorithm for the ADSL2+ and VDSL downstream lines. In Fig. 6, as the length of the VDSL line increases, the data rate region under a fixed length of ADSL2+ line decreases

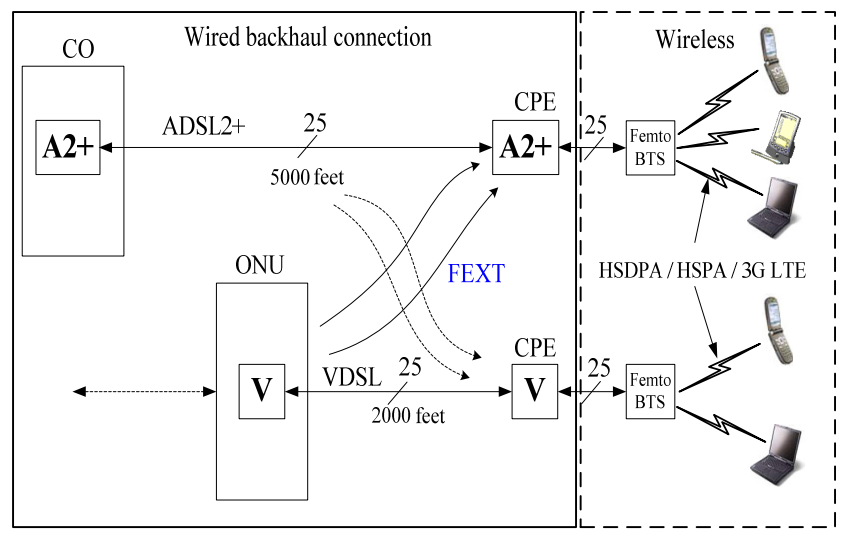

Fig. 5. CO-based ASDL2+ versus ONU-based VDSL

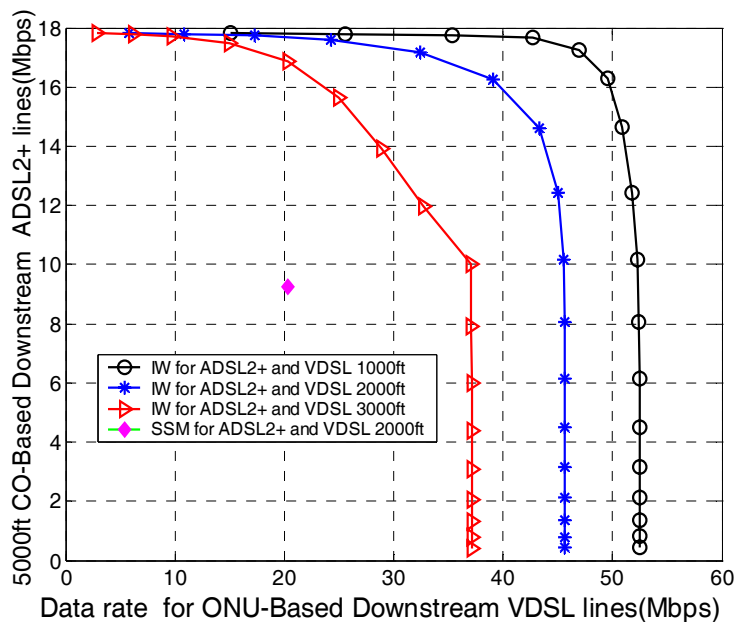

Fig. 6. Rate region in downstream fixed ADSL2+ lines and variable-length VDSL lines

due to the wide scope of the cross-talk between the ADSL2+ lines and the VDSL lines. In fact, there is a data rate tradeoff between ADSL2+ and ONU-based VDSL.

Table 1 shows the obtained data rates and the minimized power allocation for each set of lines based on the given target rates. The data rates for each set of 25 lines are the same, thus the data rates in Table 1 represent the common data rates for the 25 short and long lines. Applying the fixed margin IW algorithm, it is clear that an approximate target rate can be obtained, and that the minimized power can be obtained accordingly as long as the target rates remain within the rate region.

Table 1. Obtained data rates and minimized power allocation for each set of lines based on the given target rates for vdsl and adsl2+ downstream deployment

\begin{tabular}{c|c|c|c|c|c}
\hline \multicolumn{2}{c|}{$\begin{array}{c}\text { Target Rate } \\
\text { (Mbps) }\end{array}$} & \multicolumn{2}{c|}{$\begin{array}{c}\text { Obtained Rate } \\
(\mathrm{Mbps})\end{array}$} & \multicolumn{2}{c}{$\begin{array}{c}\text { Minimized Power } \\
(\mathrm{dBm})\end{array}$} \\
\hline ADSL2+ & VDSL & ADSL2+ & VDSL & ADSL2+ & VDSL \\
\hline 17.5 & 20 & 17.68 & 20.54 & 20.4 & -21.5 \\
\hline
\end{tabular}

\subsection{RT-VDSL and CO-VDSL based Backhaul Connections}

Fig. 7 shows the channel topology for CO-based VDSL (3000ft) versus RT-based VDSL with 50 lines in a binder. The simulation parameters are similar to those of subsection A, except that the maximum transmission power for the $\mathrm{CO}$ modems and the RT modems are $11.5 \mathrm{dBm}$ and 14.5 $\mathrm{dBm}$ [9], respectively. In addition, the North American 998 frequency plan is used to separate upstream and downstream. We assume that a fixed margin is $6 \mathrm{~dB}$ and a coding gain is $5.5 \mathrm{~dB}$ 


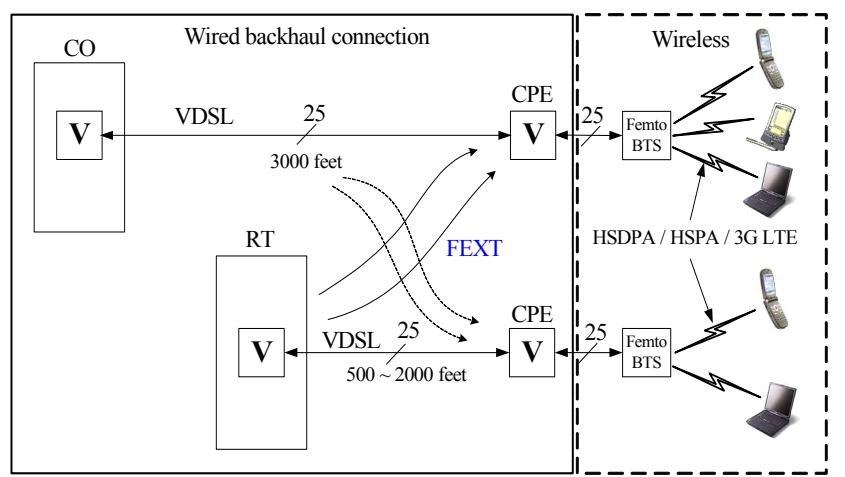

Fig. 7. Co-based VDSL (3000ft) versus RT-based VDSL(500 $\sim 2000 \mathrm{ft}$ )

One concern raised by service providers regarding the potential deployment of CO-distributed VDSL is that COdistributed VDSL has a serious downstream FEXT into RT distributed VDSL. In fact, the data rate which received by VDSL users from CO decreases dramatically without power control. This section investigates some of the trade-offs between CO-based VDSL and RT-based VDSL when using the fixed-margin IW algorithm.

Fig. 8 shows the achievable fixed-margin IW VDSL downstream rate regions for the channel topology shown in Fig. 7 For example, rates of $58 \mathrm{Mbps}$, 49.5 Mbps and 42 Mbps can be obtained for RT-based VDSL at $500 \mathrm{ft}, 1000 \mathrm{ft}$ and $2000 \mathrm{ft}$, respectively, when the $3000 \mathrm{ft}$ CO-based VDSL transmits at $25 \mathrm{Mbps}$. According to the rate regions, service providers can provide various kinds of adaptive data rates on the same binder.

In addition, the obtained data rates and minimized power allocations for each set of users are given in Table 2 based on the given target rates. RT-based VDSL is also equal to 1000 feet in this case. We can obtain the desired rate to an approximate extent and, accordingly, the minimized power can also be obtained.

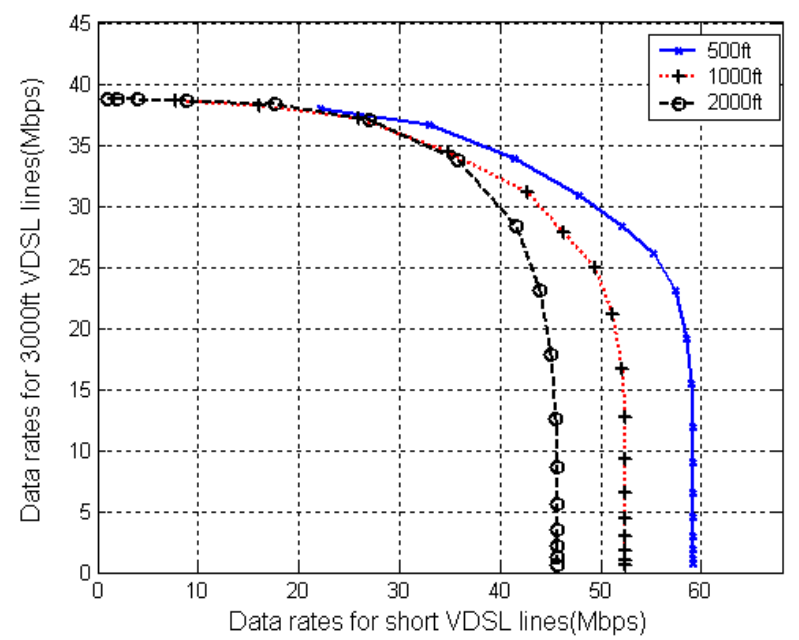

Fig. 8. Rate regions in VDSLs: 3000ft VDSL lines versus variable- length VDSL lines
Table 2. Obtained data rates and minimized power allocations for each set of users based on the given target rates for the VDSL RT/CO downstream FEXT problem.

\begin{tabular}{c|c|c|c|c|c}
\hline \multicolumn{2}{c|}{$\begin{array}{c}\text { Target Rate } \\
(\mathrm{Mbps})\end{array}$} & \multicolumn{2}{c|}{$\begin{array}{c}\text { Obtained Rate } \\
(\mathrm{Mbps})\end{array}$} & \multicolumn{2}{c}{$\begin{array}{c}\text { Minimized Power } \\
(\mathrm{dBm})\end{array}$} \\
\hline $3000 \mathrm{ft}$ & $1000 \mathrm{ft}$ & $3000 \mathrm{ft}$ & $1000 \mathrm{ft}$ & $3000 \mathrm{ft}$ & $1000 \mathrm{ft}$ \\
\hline 20 & 50 & 20.34 & 51.18 & 0.5 & -7.5 \\
\hline
\end{tabular}

\section{Conclusion}

We propose an efficient method of providing a highquality mobile streaming service to mobile users at home by maximizing the user's utility in the femtocell. The critical contribution lies in the introduction of the utility function. We derive the utility function by applying service cost, video quality and data rate. Based on the concept of user utility, we examined the rate and power control problem in the DSL-based backhaul connections, which consist of a either a mixed system composed of a downstream ONUbased VDSL and an ADSL2+, or of an RT/CO VDSL scenario for the femtocell networks. Based on competitive optimality, the proposed fixed-margin iterative water-filling algorithm enabled the lines to negotiate the best use of power and frequency with each other. Without centralized control, it is easier to implement in practice, while the minimized power constraints for multi-user modems can be obtained in order to approximately satisfy the given target rate in the femtocell. Moreover, this method is also capable of supporting an flexible data rate for different user requirements, which is determined by the data rate regions using different combinations of backhaul connections in the femtocell. In our future work, we will extend the scope of our study to the provision of diverse multimedia traffic flows for each mobile user simultaneously through the femtocell in the home.

\section{Acknowledgement}

This research was supported by the MKE (Ministry of Knowledge Economy, Korea, under the ITRC (Information Technology Research Center) support program, and was supervised by the IITA (Institute of Information Technology Assessment) (IITA-2008-C1090-0801-0038).

\section{References}

[1] "The Revolution of the Third Screen", October, 2006.

[2] Claussen, H,. "Performance of Macro and co-channel Femtocells in a Hierarchical Cell Structure", In Proc. of IEEE PIMRC, Sept., 2007. 
[3] Ho, L.T.W., Claussen, H., "Effects of User-Deployed, Co-Channel Femtocells on Call Drop Probability in a Residential Scenario", In Proc. of IEEE PIMRC, 2007.

[4] "White paper: ADSL2 and ADSL2plus - The New Standards", DSL Forum, March, 2003.

[5] Y. Liu and Z. Su, "Distributed dynamic spectrum management for digital subscriber lines", IEICE Trans. Commun., Vol.E90-B, No.3, pp.491-498, March, 2007.

[6] Li-Yu OU and Y. Chen, "An iterative multi-user bit and power allocation algorithm for DMT-based systems", IEICE Trans. Commun., Vol.E88-B, No.11, pp.42594265, Nov., 2005.

[7] W. Yu and R. Lui, "Dual methods for non-convex spectrum optimization of multi-carrier systems", IEEE Trans. Commun., Vol.54, No.7, pp.1310-1322, July, 2006.

[8] S. Panigrahi, Y, Xu, and T. Le-Ngoc, "Multi-user margin optimization in digital subscriber line channels", IEEE J. Sel, Areas Commun., Vol. 24, No.8, pp.15711580, Aug., 2006.

[9] W. Yu, G. Ginis, and J.M. Cioffi, "Distributed Multiuser power control for digital subscriber lines", IEEE J.Sel, Areas Commun., Vol.20, No.5, pp.1105-1115, June, 2002.

[10] W. Yu, G. Ginis, and J.M. Cioffi, "An adaptive multiuser power control algorithm for VDSL", In proc. of IEEE GLOBECOM, 2001.

[11] "Spectrum management for loop transmission systems", ANSI Std. T1.417, Issue 2, 2003.

[12] K.B. Song, S.T. Chung, G. Ginis, and J.M. Cioffi, "Dynamic spectrum management for next-generation DSL systems", IEEE commun. Mag., Vol.40, No.10, pp.101-109, Oct., 2002.

[13] T. Starr, J.M. Cioffi, and P.J. Silverman, Understanding Digital Subscriber Line Technology, Prentice Hall, 1999.

[14] Matthias Falkner, Michael Devetsikiotis, Ioannis Lambadaris, "An overview of Pricing Concepts for Broadband IP Networks", IEEE Communication Surveys, Second Quarter 2000.

[15] Luiz A. Dasilva, "Pricing for a QoS-Enabled Network: Survey", IEEE Communication Survey, Second Quarter, 2000.

[16] "Method for the subjective assessment of the quality of television pictures", International Telecommunication Union Radio Communication Sector, 2000.

[17] Shutto, N., Handa, Y., Higashino, T., Tsukamoto, K., Komaki, S. "Measurements of a Utility Function for Video Download Service and its Application to Service Management", In proc. of IEEE WCNC 2007.

[18] "Very-high bit-rate digital subscriber lines (VDSLs): Metallic Interface", ANSI T1E1.4/2003-210R2.

[19] Andy Tiller, "The case for Femtocells", IP.access White paper, June, 2007.

[20] http://hspa.gsmworld.com

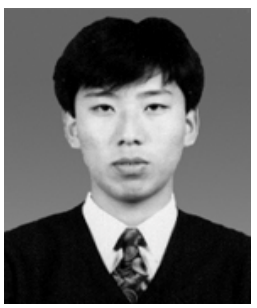

\section{Shan Guo Quan}

He received BS degrees in Electronic Engineering and Computer Science from Yanbian University of Science and Technology, China, in 1998, and an MS degree in Control and Instrumentation Engineering from Kangwon National University, Korea, in 2002. He is currently working towards a $\mathrm{PhD}$ in the Department of Electrical and Electronic Engineering, Yonsei University, Korea. His research interests include wireless multimedia communication, home networks, wireless sensor networks, and cooperative wireless networks.

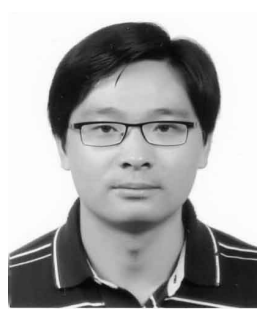

\section{Jian Xu}

He received a BS degree in Electronics Engineering from South-Central University for Nationalities, China, in 2002, and an MS degree in Electronic Engineering from Chonbuk National University, Korea, in 2004. He received a $\mathrm{PhD}$ in the Department of Electrical and Electronic Engineering, Yonsei University, Korea, in 2009. His research interests include multi-carrier transmission, resource allocation, multiple-antenna signal processing and adaptive modulation for wireless communication systems.

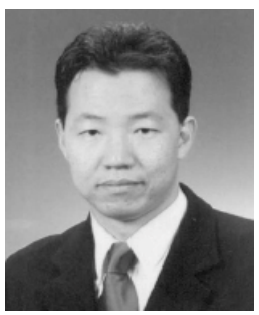

\section{Young Yong Kim}

He received his BS and MS degrees at the Department of Electronics Engineering of Seoul National University, Korea, in 1991 and 1993, respectively. He received a $\mathrm{PhD}$ in the Department of Electrical and Computer Engineering, University of Texas, Austin, USA, in 1999. From 1998 to 2000, he was employed with the Wireless Network Management Research Group at Telcordia Technologies (Formerly Bell Communication Research), Red Bank, NJ, USA, as a research Scientist. In 2000, he joined Yonsei University, Seoul, Korea, where he is currently an Associate Professor in the Department of Electrical and Electronic Engineering. His interests include wireless channel modeling, performance evaluation of mobile networks, and design and analysis of multimedia mobile communication. 